\title{
Carrying Capacity of the Environment
}

Cang Hui, Centre for Invasion Biology, Department of Mathematical Sciences, Stellenbosch University, Matieland, South Africa; and African Institute for Mathematical Sciences, Muizenburg, South Africa

(c) 2015 Elsevier Ltd. All rights reserved.

This article is a revision of the previous edition article by M.E. Geores, volume 10, pp. 7038-7039, (c 2001, Elsevier Ltd.

\begin{abstract}
Catton defines carrying capacity as the maximum persistently supportable load of a focal population in a bounded environment. Here, definitions of carrying capacity for natural populations, ecosystems and humans, as well as biocapacity, are presented, with issues fully discussed pertaining to their proper interpretation, models and estimation, debate and derived paradoxes. With the explosion of the human population, the threat of overstepping the earth's carrying capacity has become a sword of Damocles, hanging over humanity and urging it to choose a sustainable path of development. Carrying capacity has become arguably the most important concept in the era of Anthropocene.
\end{abstract}

Humans are fascinated by growth dynamics, from the weight of an infant to the size of an economy. Two intrinsic questions on any growth dynamics are how fast and how long the system can grow. That is, the rate and the bound of growth. An unbounded growth is infeasible due to limited resources, and carrying capacity is a measure of this limitation. In 1798, Thomas Malthus proposed an exponential growth curve in his Essay on the Principle of Population, stating that a population with constant fecundity greater than its mortality will grow exponentially till it causes inevitable conflicts with the limited supply of resources. Deminishing resources and the expanding population will inevitably lead to scramble competition. Malthus's idea has further inspired Charles Darwin to adopt resource competition as one prerequisite of evolution via natural selection. Darwin said in his Autobiography that, "I happened to read for amusement Malthus on Population and being well prepared to appreciate the struggle for existence which everywhere goes on from long-continued observation of the habits of animals and plants, it at once struck me that under these circumstances favourable variations would tend to be preserved, and unfavourable ones to be destroyed. The result of this would be the formation of new species." Such struggle of life will ensure only the survival of the fittest, or equivalently as Russel Wallace put, the elimination of the unfit. Such a density-dependent mechanism of population regulation that intense competition happens when resource demand approaches supply was formally formulated by Pierre Verhulst in 1838 , by adding a parameter $K$ into the Malthusian equation to curb the unbounded growth. This equation is now well-known as the logistic equation, and the parameter $K$ the carrying capacity.

\section{Population Carrying Capacity}

Catton (1986), defined an environment's carrying capacity as its maximum persistently supportable load. In ecology, carrying capacity is normally defined as the maximum population of a given species that can be supported indefinitely in a defined habitat without permanently impairing the productivity of that habitat (Rees, 1992). The concept of carrying capacity is rooted in the logistic equation depicting population dynamics under simple density-dependent regulation and has been developed across different hierarchical levels of living systems and in many sectors of human society (Seidl and Tisdell, 1999; Monte-Luna et al., 2004). In the Malthusian equation, the probability that an organism will reproduce or die is assumed to remain constant and is independent of the density of the population. Obviously, this can only be true when there is no competition among the individuals, normally when the population density is low. The growth of any population in a limited environment will eventually halt due to the shortage of resources. When the demand of the existing population on the resource (i.e., the population size times the basic per capita resource consumption rate required for maintaining life) is equal to the rate of resource supply, the population will reach its saturation level. This saturation level is decided by both the resource supply and the per capita resource consumption rate, called the carrying capacity of the environment for the focal population. Using the language of differential equations in mathematics, the Verhulst logistic equation reads as follows:

$$
\frac{d N}{d t}=r N\left(\frac{K-N}{K}\right)
$$

where $N$ is the population size; $d N / d t$ the derivative of population size, indicating the change rate of the population size; $r$ the intrinsic population growth rate, indicating how fast the population can grow at small size; $K$ the carrying capacity. The Malthusian equation only includes the first part of the right term, $d \mathrm{~N} / d t=r \mathrm{~N}$, meaning that the population change rate is proportional to its current population size. It also means that the per capita rate of increase $(d N / d t) / N$, often an indicator of population fitness, is a constant and thus density independent. In the logistic equation, the per capita rate of increase, $r(K-N) / K$, is a declining function of current population size $N$, thus becoming density dependent. In Figure 1, we can clearly see the difference between Malthusian's exponential growth curve and the saturation form of the logistic equation. Clearly, when $K$ approaches infinity, the logistic equation becomes the 


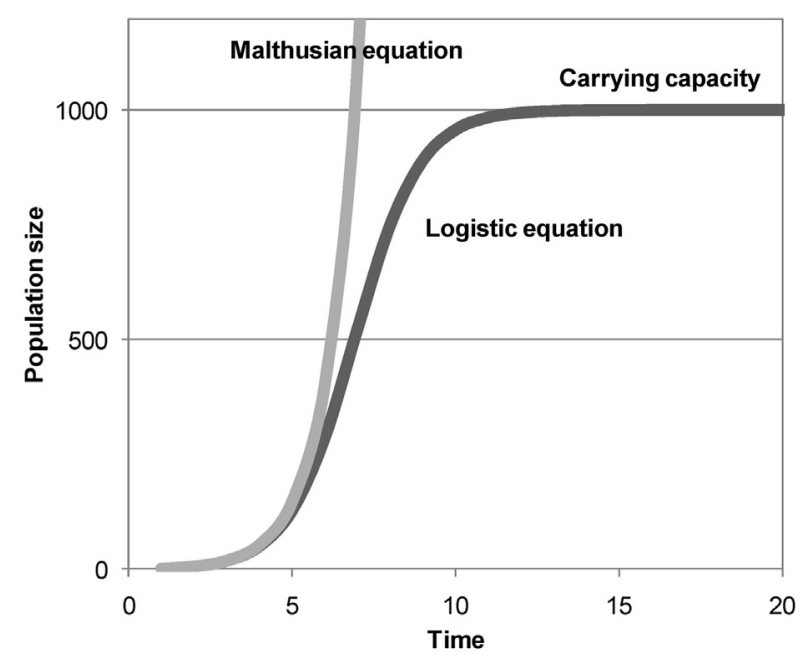

Figure 1 An illustration of population dynamics governed by the Malthusian equation and the logistic equation. Parameter values: $r=N_{0}=1$ and $K=1000$.

Malthusian equation. The exact solution to the logistic equation exists,

$$
N(t)=\frac{K \cdot N_{0} \cdot \exp (r \cdot t)}{K+N_{0}(\exp (r \cdot t)-1)}
$$

where $N_{0}$ is the population size when time $t=0$. When time $t$ increases, the population size will converge to the carrying capacity $K$ (Figure 1 ). The logistic equation is the simplest model, which contains a carrying capacity for the population. In fact, carrying capacity exists in many models that implement the negative density-dependence term, i.e., $(d N / d t) / N$ is a declining function of $N$.

Because the carrying capacity is also the asymptote, or the equilibrium of the population size in the logistic equation, the concept of carrying capacity has often been confused with population equilibrium, leading to unnecessary confusion and so-called paradoxes. In practice, many studies have considered the maximum population equilibrium or maximum population size as the carrying capacity, making the distinction even muddier. In 1969, Richard Levins hypothesized that if $r$ in the logistic equation is negative, then we will have a positive $d N / d t$ when $N>K$ and thus the population size will increase indefinitely. This is clearly counterintuitive. Although constraining $r$ to be only positive can make Levins' paradox disappear, many declining populations are indeed experiencing the situation of $r<0$, thus such a constraint could exclude many biologically meaningful scenarios. Although constraining $r$ and $K$ to be the same sign could resolve Levins' paradox, such a constraint nonetheless incurs a new problem on the meaning of negative carrying capacity $(K<0)$. The negative carrying capacity could be a measure of just how unfavorable the environment is (Hui, 2006). An important finding from metapopulation theory is that the source-sink dynamics are such that where a local population is in a 'sink habitat' a positive equilibrium can still be maintained due to contributions from migrants from source populations. These sink patches have a negative carrying capacity but support a positive population equilibrium. Clearly, the carrying capacity of a sink habitat is not equal to the maximum population equilibrium that can survive through the inflow of propagules, known as the rescue effect. This highlights the difference between carrying capacity and the maximum population equilibrium. Indeed, many preindustrial cities often benefited from being the hub of trade routes and supported a large population beyond their capacity.

In 1992, Lev R. Ginzburg considered incorporating additional mortality into the logistic equation without changing any other aspects of the environment. One of his proposals is: $d N / d t=r N(1-N / K)-\mu N$, where $\mu N(\mu>0)$ is the additional mortality term. As the resources do not change in this hypothetical scenario, intuitively the population with higher mortality might attain the same $K$, but only slower. However, the new population equilibrium is $K(r-\mu) / r(\mu<r)$, lower than the carrying capacity $K$. Once again, the Ginzburg paradox is due to the confusion between the concept of carrying capacity and population equilibrium. To illustrate, let us consider a thought experiment (Hui, 2006). There are three eggs in a six-position egg box (Figure 2). If we eat one and then put a new one in it everyday, the size of the egg population will be maintained at three. Now what is the carrying capacity of this egg population, three or six? Now, if we eat two eggs per day, the population equilibrium will become zero but the carrying capacity remains six. According to our knowledge, the above equation does not have any mechanistic problem but has been widely used as a theoretical foundation in spatial and metapopulation ecology. This new stable equilibrium is called the local carrying capacity of a metapopulation, whereas other scientists call this equilibrium local density. Once again, the concept of equilibrium was confused with carrying capacity. This confusion also appears in some textbooks, yet many others do differentiate between these two concepts. The carrying capacity of the egg box example should be the 'environment's maximal load,' i.e., the six positions in the egg box, and the equilibrium of egg population is three if we eat one and add one per day. If we can clarify these two concepts: carrying capacity and population equilibrium (Hui, 2006), there is no contradiction between intuition and there should be no Ginzburg's paradox.

Ecological carrying capacity is a measure of the amount of renewable resources in the environment in units of the number of organisms these resources can support. Normally, $K$ is a function of both the species and the environment, and is expected to change only in evolutionary time (Daily and Ehrlich, 1992).

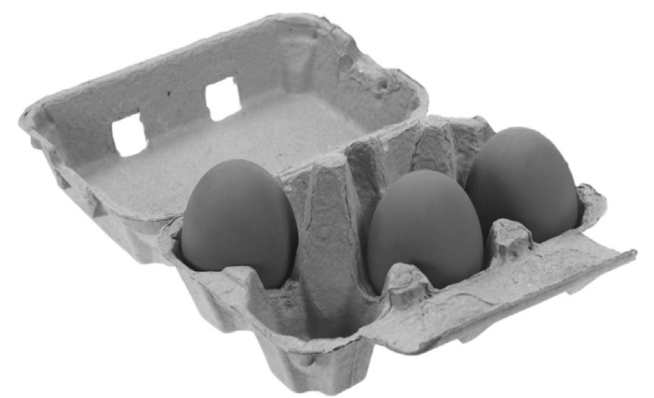

Figure 2 A population of three eggs at the equilibrium in an egg box with the six-holder carrying capacity. 
Rees (1992) suggested that carrying capacity is the number of individuals of a given species that a given habitat can support without being permanently damaged. If the population of a given species exceeds the carrying capacity of a given habitat, then either the resources required to meet the needs of that species will be depleted, or the wastes produced by that species will build to the point of poisoning members of the species, or both, and the population will crash. In livestock management, carrying capacity is affected by many factors, such as habitat, climate, vegetation, soil profile, food quantity and quality and accessibility, inter- and intracompetition, foraging, parasitism, diseases, population density, social behavior, and anthropogenic disturbance. In terms of nutrition intake, carrying capacity has been defined as the accessible and utilizable energy in the habitat divided by per capita energy consumption (Ayllon et al., 2012). In terms of space, carrying capacity is also affected by aggregation or social group behavior, group size, dispersal distance, and habitat suitability, and can be defined as the amount of habitat available divided by the expected individual territory area for a given life stage (Ayllon et al., 2012). It is closely related to subsistence density, tolerance density, security density, maximum harvest density, minimum impact density in livestock and wildlife management (Hobbs and Hanley, 1990).

Carrying capacity is not a static number but is affected by the abundance and distribution of limited resources and by how individuals compete for these limiting resources (Ayllon et al., 2012). This notion is especially relevant in organisms that compete via both exploitation and interference because of behavioral responses, such as competition avoidance, induced by aggressive interactions typically result in a much reduced exploitation of the limited resource than could be accounted for by resource depletion alone (Ayllon et al., 2012). In territorial species, the behavioral adjustment of the size and shape of territories has profound consequences for their population regulation, demography, and spatial ecology. In practice, carrying capacity is determined by the resource in the least supply, 'the weakest link' as it were. For production purposes, accurate estimation of carrying capacity will affect the maximum sustainable yield, which is thought to be obtained when the population is maintained at half of the carrying capacity (Ayllon et al., 2012). By contrast, in conservation efforts, the maximum carrying capacity is the desired target.

\section{Carrying Capacity of Ecosystems}

The directionality of community succession is a powerful concept for conservation biology, analogous to the irreversibility of time in physics, and has revolutionized the understanding of complex adaptive systems. By definition, succession is 'an orderly process of community change' after disturbance. Knowing the directionality of succession is necessary for (1) distinguishing new from mature communities (i.e., defining the age of a community), (2) understanding how communities evolve and respond to disturbance (e.g., habitat loss and climate change), and (3) designing more efficient conservation and restoration plans. Two important concepts of an ecosystem are productivity (often measured by the total biomass) and biodiversity (often measured by species richness or the Shannon index that considers the relative abundance of species). The directionality of succession can potentially be indicated by the increase of net primary production. Per capita community productivity can be very high during the initial phases of succession, and decreases progressively as an upper limit to biomass is reached, and this phenomenon may be expressed as a sigmoid curve roughly akin to the logistic growth curve of a population. The upper limit of net primary production, or the maximum potential biomass, has been suggested to indicate the carrying capacity of biomass in a community (Monte-Luna et al., 2004).

If the number of species in a habitat is relatively low, immigration and diversification through disruptive selection will take place, which progresses until the region attains a maximum supportable load of species (Monte-Luna et al., 2004). This can also define the directionality of succession and, thus, the carrying capacity of biodiversity, depicting the maximum number of species or the 'biodiversity ceiling' that an environment can support. In fact, the form of species diversification on evolutionary timescales resembles the logistic population growth. By analogy, the number of species resembles the number of individuals or biomass, and the difference between rate of speciation and extinction the difference between fecundity and mortality (i.e., the intrinsic growth rate). The carrying capacity of biodiversity may be regulated by both physical factors such as climate and habitat heterogeneity and biotic interactions between species. Once the biodiversity reaches its ceiling, interspecific interactions can lead to competitive exclusion of some taxa, and adaptive niche partitioning (Monte-Luna et al., 2004). This can be further related to the $r / K$ selection theory in ecology. It specifies a lifehistory tradeoff between the values of $r$ and $K$ that a species can possess. Species with high $r$ and low $K$ are opportunistic and often occur at the early stage of succession, whereas species with low $r$ and high $K$ are good competitors for limiting resources and often occur at the late stage of succession. An intermediate level of disturbance in a landscape, arguably, can create patches at different levels of succession, thus promoting the coexistence of these two types of species at the regional scale.

\section{Human Carrying Capacity}

Our planet is practically a closed system in terms of physical resources, and all human activity depends on these limited resources. Due to the continue growing of the world population and the rapid diminishing of pristine ecosystems from our exploration for consumption, the concept of carrying capacity is essential and should be considered paramount in our future planning. The Club of Rome has warned us the possible consequences of world population growth and industrialization for food production and resource exhaustion. They projected that within 50 years from now, the planet would reach its limit of growth (Figure 3), and thus advocated an urgent mutually beneficial integration of economics and ecology. Approaching carrying capacity means the deterioration of ecosystem services, the loss of biodiversity and habitat heterogeneity and the break down 


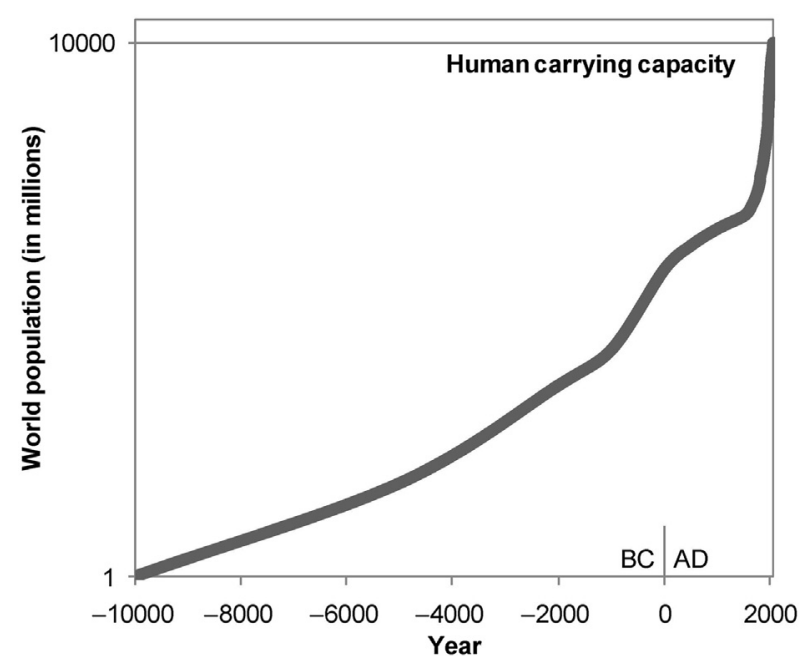

Figure 3 World human population from $10000 \mathrm{BC}$ to AD 2050 when the carrying capacity of 10 billion is projected to be reached.

of ecosystem resilience (Arrow et al., 1995). This could lead to irreversible change, enlarged uncertainty to the biosphere, and even a regime shift of the world's living and climate systems. This concern has greatly influenced the popularity of environmentalism and the creation of the United Nations Environment Program.

Carrying capacity is commonly assumed to be constant in population dynamics models used for resource management. Human carrying capacity, however, is not a fixed, static, or a simple relationship, but is dynamic and adaptive. Humans can have more than one $K$ value for a given resource at a given time, and the carrying capacity can vary markedly with culture and level of economic development (Daily and Enrlick, 1992). Earth's human carrying capacity cannot be characterized by a simple monotonic decline of the natural capital as the number of humans increase. For instance, Cohen (1995) studied a dynamic $K$ model, with the change of carrying capacity affected by the population change rate.

Human carrying capacity describes the number of human beings that can be supported on a sustainable basis in a given area (or on the whole Earth) within natural resource limits and by human choices concerning social, cultural, and economic conditions (Franck et al., 2011). This concept is twofold: Biophysical carrying capacity is the maximum population that can be supported by the resources of planet Earth at a given level of technology, whereas social carrying capacity is the sustainable biophysical carrying capacity within a given social organization (Franck et al., 2011). In the case of very efficient agriculture the carrying capacity is only determined by the ratio of the total productive area and the nonagricultural used area (per person). For 2005 land cover, it can support 11.4 billion $\left(10^{9}\right)$ (Franck et al., 2011), ranging from 7.7 to 12 billion in literature (Cohen, 1995). It has been predicted to be much higher than this range under certain scenarios, ranging from 30 to 100 billion (Franck et al., 2011). It is the limiting resource that constrains human carrying capacity. Although there exists the technology and economic willingness to develop intensive sustainable agriculture and other forms of food supply compatible with the aims of world development programs.

In a Malthusian framework, economic growth generates population growth; the population growth increases pressure on the natural resource base; the deterioration of the natural resource base decreases per capita food output; and the reduced per capita food limits population growth which, in turn, limits exploitation of the environment (Berck et al., 2012). This moderating feedback loop (density dependence) can be diverged by technological improvements. Population pressure on the resource base stimulates technological improvements in food production and hence facilitates continued population growth, which intensifies exploitation of the resource base (Berck et al., 2012). Alternatively, as income goes up there is increasing environmental degradation up to a point, after which environmental quality improves, causing an invertedU shape (Arrow et al., 1995). Moreover, the detrimental impact from human society to ecosystems is often discounted over time and distance in decision-making, i.e., discounting future and faraway activities than can reduce carrying capacity. This encourages behavior that may reduce carrying capacity for future generations (Daily and Enrlick, 1992). Globalization further creates the illusion that all regions could simultaneously sustain populations that sum to more than global $K$, a typical Netherlands fallacy.

\section{Biocapacity}

Since the concept of sustainable development was put forward, it has become an ideal development mode and a common policy goal. To date, many indicators have been developed to assess the status of sustainable development, such as the lifecycle assessment, human development index by the United Nations Development Programme, barometer of sustainability, index of sustainable economic welfare, environmental pressure indicator, genuine progress indicator, sustainable technology development, environmental sustainability index, and ecological footprint (Rees, 1992). The latter has gained popularity due to its compatibility with the data format commonly derived from economic and social surveys. The ecological footprint for a particular population is defined as the total area of productive land and water ecosystems required to produce sufficient resources and assimilate wastes (Rees and Wackernagel, 1994). Biocapacity can be defined as the locally available carrying capacity of the ecosystem for generating resources and absorbing wastes (Rees and Wackernagel, 1994) and is constrained by the carbon sequestration rate of the ecosystem. According to the global average rate of carbon sequestration, we need to have about 17 ha of land and ocean area to absorb 1 metric ton of carbon emission. Ecological footprint and biocapacity, thus, represent the demand on and the supply from a regional ecosystem, respectively. As both ecological footprint and biocapacity are measured in the same unit (the global hectare: gha), it is straightforward to calculate regional ecological budget as surplus and deficit, after discounting the influence of trading. This specifies whether a regional population is potentially self-sufficient or is at least partially reliant on imported biocapacity. An ecological surplus has been proposed as a minimum criterion for sustainability. 
Due to it being highly operable and easy to understand for public and policy makers, with the data required accessible from government yearbooks, to date they have been applied at a variety of spatial scales, from municipality/provincial level to national/global extents, covering all aspects of socioeconomic sectors, such as industry, education, agriculture, tourism, and waste management.

As a continuously developing field, the ecological footprint methodology has been widely criticized and amended. For instance, it has been considered a static indicator of weak sustainability as no dynamics and bounds are imposed on the level of ecosystem services and their demands. This has been partially solved by time series analysis and extrapolation (Yue et al., 2013). The introduction of spatial features, with the help of the geographic information system (GIS), has largely released the methodology from this constraint. To calculate the biocapacity of a region, one first needs to estimate the available areas of biologically productive land and water. Specifically, this biologically productive area can normally be divided into six or more main categories (cropland, grazing land, fishing land, forest, built-up area, and barren land), and the sizes of these six land covers can then be either extracted from government agencies or calculated using remote sensing images with the aid of GIS (Yue et al., 2013):

$$
K=\sum_{i} A_{i} \cdot Y_{i} \cdot E_{i}
$$

where $A_{i}$ is the biologically productive area of land cover category $i ; y_{i}$ is the yield factor of land category $i$ and is calculated annually as the ratio of the local yield of a generic product to the global average yield of the same product. The yield factor converts local biologically productive land into units of global average productivity and thus facilitates comparisons across regions; $E_{i}$ represents the equivalence factor of land cover category $i$ and is a scaling factor needed for converting a specific land use type into a universal unit of biologically productive area (gha). Equivalence factor is calculated each year as the ratio of the global average productivity of a specific land type to the average productivity of all biologically productive land on the earth.

An important issue that is associated with area-based information is the scale dependency of spatial features. Evidently, the shape and size of different land covers are sensitive to the spatial scale as most landscape features are scale dependent and have self-similar, fractal structures. Put simply, results for biocapacity can be influenced by the resolution of the map (Yue et al., 2013). Since area-based information has been widely implemented for estimating the sizes of different land covers and therefore the biocapacity, it is important to at least be aware of the scale dependency of biocapacity and its impact on regional sustainability assessment (Figure 4). Moreover, there is often a power-law relationship between the biocapacity and population density (Figure 5), suggesting that biocapacity serves not only as a support for social development and human well-being but also sets an ecological limit for human activities (Yue et al., 2011). The biocapacity pressure index is the regional ecofootprint to biocapacity ratio and reflects the pressure posed by the human population on the local ecosystem. If the biocapacity pressure index is greater than one, the population is in ecological

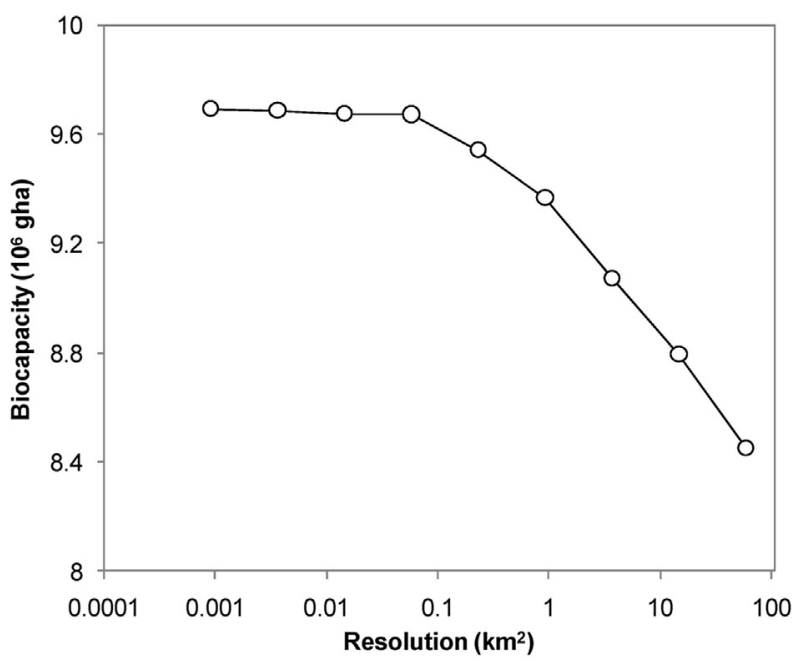

Figure 4 The scale dependency of the biocapacity in Jinghe River Watershed, Northwest China.

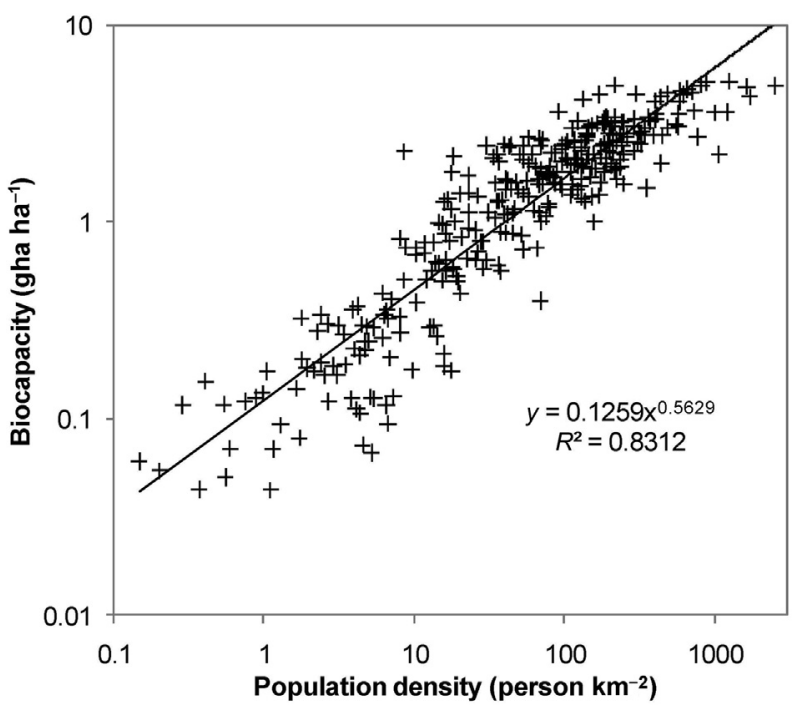

Figure 5 The relationship between population density and biocapacity per hectare for 320 counties in Northwest China.

overshoot, indicating that regional development is not ecologically self-sustainable. If the biocapacity pressure index is less than one, the region is potentially self-sufficient (Yue et al., 2011).

\section{Relevance in Anthropocene}

The growing number of studies on carrying capacity and its further development into different research fields have made this concept the most important issue in this century, for obvious reasons. First, the world has entered a rather peaceful era after the World War II, allowing both the living condition to proliferate and the economy to boom at a staggering pace. Although it took the modern human population of Homo 
sapiens 200000 years to reach its first billion in the early nineteenth century, the second billion took only another 100 years. Standing at 7 billion currently, the human population is projected to reach 10 billion in the middle of this century. The sheer number and the increasing per capita consumption rate in this era of Anthropocene place an enormous pressure on the planet's resource supply, both renewable and unrenewable. Humanity as a single species consumes more than one-fifth of the planet's net primary production each year. Will this great era of modern humanity continue to grow indefinitely; fluctuate around a sustainable level after a soft landing; or follow the boom-bust pattern of many earlier civilizations? The concept of carrying capacity has become a great reminder, a type of 'sword of Damocles,' hanging over humanity and driving it onto a sustainable path.

Second, as human population continues to expand, the planet's pristine ecosystems are degrading at a rapid rate due to man-made global environmental changes - climate change, habitat loss, biological invasion, soil erosion, and pollution, to name a few. Ongoing agricultural intensification, overfishing, industrialization, and urbanization further transfer how seminatural ecosystems function and behave, creating many novel ecosystems that face high disturbance and gene flow. The planet's greatest asset - biodiversity, 100 million species strong is quickly losing its grip. The rapid loss of species is estimated to be between 1000 and 10000 times higher than the natural extinction rate, reaching 10000 species extinction per year many of which disappear before they have even been formally described. Human society relies heavily on the health of ecosystem functions, the provision of ecosystem services and the benefit from biological diversity for cleaning air and water, stabilizing weather, maintaining soil fertility, dissipating waste, controlling pests, pollinating crops, generating power and discovering new antibodies, and providing food, timber, cloth, medicine, industrial material (coal, oil, gas, rubber, plastics, chemicals), together with minerals. We are not making anything new but utilizing products and services of the planet's ecosystem and physical system. To sustain humanity, we need to manage the planet's biosphere within its bearable margin to avoid disruptive regime shift and massive extinction, although some argue that we have already crossed the point of no return. On the positive side, the concept of carrying capacity is a powerful tool for assessing and conserving natural capital.

See also: Environment and Development; Environmental Movements; Food Security and 'Green Revolution'; Limits to Growth; Sustainable Development: An Economic Perspective.

\section{Bibliography}

Arrow, K., Bolin, B., Constanza, R., Dasgupta, P., Folke, C., Holling, C.S., Jansson, B.O., Levin, S., Maler, K.G., Perrings, C., Pimentel, D., 1995. Economic growth, carrying capacity, and the environment. Science 286, 520-521.

Ayllon, D., Almodovar, A., Nicola, G.G., Parra, I., Elvira, B., 2012. Modelling carrying capacity dynamics for the conservation and management of territorial salmonids. Fisheries Research 134-136, 95-103.

Berck, P., Levy, A., Chowdhury, K., 2012. An analysis of the world's environment and population dynamics with varying carrying capacity, concerns and scepticism. Ecological Economics 73, 103-112.

Catton, W., 1986. Carrying Capacity and the Limits to Freedom. Paper Prepared for Social Ecology Session 1. XI World Congress of Sociology, New Delhi, India.

Cohen, J.E., 1995. Population growth and Earth's human carrying capacity. Science 269, 341-346.

Daily, G.C., Ehrlich, P.R., 1992. Population, Sustainability, and Earth's Carrying Capacity. Paper No. 0046. Department of Biological Sciences, Stanford University, Stanford.

Franck, S., von Bloh, W., Muller, C., Bondeau, A., Sakschewski, B., 2011. Harvesting the Sun: new estimations of the maximum population of planet Earth. Ecological Modelling 222, 2019-2026.

Hobbs, N.T., Hanley, T.A., 1990. Habitat evaluation - do use availability data reflect carrying capacity. Journal of Wildlife Management 54, 515-522.

Hui, C., 2006. Carrying capacity, population equilibrium, and environment's maximal load. Ecological Modelling 192, 317-320.

Monte-Luna, P.D., Brook, B.W., Zetina-Rejon, M.J., Cruz-Escalona, V.H., 2004. The carrying capacity of ecosystems. Global Ecology and Biogeography 13, 485-495.

Rees, W.E., 1992. Ecological footprint and appropriated carrying capacity: what urban economics leaves out. Environmental Urbanization 4, 121-130.

Rees, W.E., Wackernagel, M., 1994. Ecological footprints and appropriated carrying capacity: measuring the natural capital requirements of the human economy. In: Jansson, A.M., Hammer, M., Folke, C., Constanza, R. (Eds.), Investing in Natura Capital: The Ecological Economics Approach to Sustainability. Island Press, Washington, DC, pp. 362-390.

Seidl, I., Tisdell, C.A., 1999. Carrying capacity reconsidered: from Mathus' population theory to cultural carrying capacity. Ecological Economics 31, 395-408.

Yue, D., Xu, X., Hui, C., Xiong, Y., Han, X., Ma, J., 2011. Biocapacity supply and demand in Northwestern China: a spatial appraisal. Ecological Economics 70, 988-994.

Yue, D., Guo, J., Hui, C., 2013. Scale dependency of biocapacity and the fallacy of unsustainable development. Journal of Environment Management 126, 13-19.

\section{Relevant Websites}

http://www.carryingcapacity.com.au/

http://www.carryingcapacity.org/

http://www.facingthefuture.org/

http://www.sustainablescale.org/ 